\title{
THE
}

\section{A Sensitivity Study of the Thermodynamic Environment on GFDL Model Hurricane Intensity: Implications for Global Warming}

Weixing Shen

University of Rhode Island

Robert E. Tuleya

Isaac Ginis

University of Rhode Island, iginis@uri.edu

Follow this and additional works at: https://digitalcommons.uri.edu/gsofacpubs

Terms of Use

All rights reserved under copyright.

\section{Citation/Publisher Attribution}

Shen, W., R.E. Tuleya, and I. Ginis, 2000: A Sensitivity Study of the Thermodynamic Environment on GFDL Model Hurricane Intensity: Implications for Global Warming. J. Climate, 13, 109-121, doi: 10.1175/ 1520-0442(2000)0132.0.CO;2.

Available at: https://doi.org/10.1175/1520-0442(2000)0132.0.C0;2

This Article is brought to you for free and open access by the Graduate School of Oceanography at DigitalCommons@URI. It has been accepted for inclusion in Graduate School of Oceanography Faculty Publications by an authorized administrator of DigitalCommons@URI. For more information, please contact digitalcommons-group@uri.edu. 


\title{
A Sensitivity Study of the Thermodynamic Environment on GFDL Model Hurricane Intensity: Implications for Global Warming
}

\author{
WEIXING SHEN \\ Graduate School of Oceanography, University of Rhode Island, Narragansett, Rhode Island \\ ROBERT E. TUleya \\ NOAA/Geophysical Fluid Dynamics Laboratory, Princeton, New Jersey \\ ISAAC GINIS \\ Graduate School of Oceanography, University of Rhode Island, Narragansett, Rhode Island
}

(Manuscript received 13 May 1998, in final form 4 February 1999)

\begin{abstract}
In this study, the effect of thermodynamic environmental changes on hurricane intensity is extensively investigated with the National Oceanic and Atmospheric Administration Geophysical Fluid Dynamics Laboratory hurricane model for a suite of experiments with different initial upper-tropospheric temperature anomalies up to $\pm 4^{\circ} \mathrm{C}$ and sea surface temperatures ranging from $26^{\circ}$ to $31^{\circ} \mathrm{C}$ given the same relative humidity profile.

The results indicate that stabilization in the environmental atmosphere and sea surface temperature (SST) increase cause opposing effects on hurricane intensity. The offsetting relationship between the effects of atmospheric stability increase (decrease) and SST increase (decrease) is monotonic and systematic in the parameter space. This implies that hurricane intensity increase due to a possible global warming associated with increased $\mathrm{CO}_{2}$ is considerably smaller than that expected from warming of the oceanic waters alone. The results also indicate that the intensity of stronger (weaker) hurricanes is more (less) sensitive to atmospheric stability and SST changes. The model-attained hurricane intensity is found to be well correlated with the maximum surface evaporation and the large-scale environmental convective available potential energy. The model-attained hurricane intensity is highly correlated with the energy available from wet-adiabatic ascent near the eyewall relative to a reference sounding in the undisturbed environment for all the experiments. Coupled hurricane-ocean experiments show that hurricane intensity becomes less sensitive to atmospheric stability and SST changes since the ocean coupling causes larger (smaller) intensity reduction for stronger (weaker) hurricanes. This implies less increase of hurricane intensity related to a possible global warming due to increased $\mathrm{CO}_{2}$.
\end{abstract}

\section{Introduction}

The effect of atmospheric (dry and moist) static stability on hurricane intensity has received little attention compared to some other factors. Change of tropical atmospheric stability may, however, be relatively large in some climate scenarios. Recently, maximum potential intensity (MPI) theories (e.g., Emanuel 1988, 1995; Holland 1997) have been developed using large-scale thermodynamic environmental factors. These theories are commonly used to estimate the effects of environmental changes on maximum achievable hurricane intensity and to provide reasonable upper limits of hur-

Corresponding author address: Dr. Weixing Shen, Physical Oceanography, Graduate School of Oceanography, University of Rhode Island, Narragansett, RI 02882.

E-mail: wshen@circe.gso.uri.edu ricane intensity (e.g., Schade and Emanuel 1999; Holland 1997). However, some factors that may influence hurricane intensity, such as asymmetries, hurricane-environment interactions, and hurricane-ocean interaction, are not typically considered in these MPI derivations. A major criticism (e.g., Lighthill et al. 1994) concerning the use of climate models to investigate the effects of environmental changes on hurricanes, particularly on hurricane intensity, is their coarse resolution. In this study, we focus on the effects of concurrent atmospheric stability and SST changes on hurricane intensity using the high-resolution Geophysical Fluid Dynamics Laboratory (GFDL) hurricane model. Rather than focusing on several specifically observed or modelsimulated vertical thermodynamic profiles (e.g., Knutson et al. 1998), we investigate the effects of SST and stability over a wide parameter space. For our approach, integrations are made starting with specified initial cyclones embedded in idealized environments of temper- 
ature lapse rate anomalies. The majority of the experiments in this study are performed with SSTs fixed in time. However, the SSTs usually decrease due to the air-sea interaction in hurricanes (Ginis 1995) which may significantly affect the hurricane intensity (Bender and Ginis 2000). Therefore, a set of additional experiments has been conducted to investigate the role of ocean coupling in hurricane intensity for different atmospheric environmental conditions. It will be interesting to compare these idealized model results with the more simple MPI theories and with the more specific GCM global warming studies.

Strictly speaking, the change of moist static stability, which combines temperature and moisture profiles in the atmosphere, is restricted since equivalent potential temperature tends to be conserved. Emanuel (1987) and Emanuel et al. (1994) suggested that tropical atmosphere is nearly convectively adjusted or neutral. The existence of a nearly neutral state or near-zero convective available potential energy (CAPE) can be seen from some tropical soundings (e.g., Betts 1982; Xu and Emanuel 1989). However, deviations from this state may occur and precise definitions of this neutral state vary. Williams and Renno (1993) analyzed various tropical experiment data sources and found that the tropical atmospheric CAPE ranges from almost zero to above 5000 $\mathrm{J} \mathrm{kg}^{-1}$ (reversible ascent was used with the inclusion of freezing at very high altitudes). Large CAPE and its change were either implied or indicated in both observations (Oort 1983; Henderson-Sellers et al. 1998) and climate model simulations (e.g., Ye et al. 1998). In this study, we will investigate the correlations of modelattained intensity with both dry stability and CAPE.

The paper is organized as follows. Section 2 gives a brief description of the National Oceanic and Atmospheric Administration/GFDL hurricane model and the experiment design in this study. Presented in section 3 are the results of our sensitivity experiments to atmospheric static stability versus SST. Some explanations and discussions about the results are made in section 4 . In section 5 a brief summary of this study is made.

\section{Model and experiment design}

\section{a. Model description}

The hurricane model applied in this study is the triply nested movable mesh model described by Kurihara and Bender (1980). The model is a primitive equation model formulated in latitude, longitude, and $\sigma$ coordinates, with 18 levels in the vertical (Kurihara et al. 1990, Table $1)$. The domains of the three meshes in this study are $75^{\circ} \times 75^{\circ}, 11^{\circ} \times 11^{\circ}$, and $5^{\circ} \times 5^{\circ}$ with resolutions of $1^{\circ}, 13^{\circ}$, and $16^{\circ}$, respectively. The outermost domain ranged from $10^{\circ} \mathrm{S}$ to $65^{\circ} \mathrm{N}$ in the meridional direction. The longitudinal positioning of this domain does not possess any specific meaning since zonally uniform environmental states are used in all the experiments in this study. The outermost domain is fixed in the model, while the inner meshes move with hurricane center. The model physics includes cumulus convection treated by the parameterization scheme of Kurihara (1973) with some additional modification (Kurihara and Bender 1980, appendix C); surface exchanges via the Monin-Obukhov scheme; subgrid-scale horizontal diffusion treated by the nonlinear viscosity scheme (Smagorinsky 1963); vertical diffusion via the Mellor and Yamada (1974) level-2 turbulence closure scheme with a background diffusion coefficient added; and the Schwarzkopf and Fels (1991) infrared and the Lacis and Hansen (1974) solar radiation parameterizations with cloud specification. The GFDL model has been implemented as an official hurricane forecast model of the National Weather Service since 1995. An overall summary of the GFDL model and forecast system can be found in Kurihara et al. (1998).

The Princeton ocean model is coupled to the hurricane model for a set of experiments in this study. This ocean model is a free surface primitive equation model in $\sigma$ coordinate system with the same vertical and horizontal diffusion schemes as used in the atmospheric model. Details of this model are described in Bender et al. (1993b) and Blumberg and Mellor (1987).

\section{b. Experiment design}

For the experiments without ocean coupling, SSTs are fixed during 3-day integrations. The initialization scheme by Kurihara et al. (1993), with some recent modifications (Kurihara et al. 1995), is applied for model initialization. Simply, a vortex of model-produced axisymmetric and $\beta$-effect related asymmetric components is superposed on an environmental field. The initial vortices are placed at $19.5^{\circ} \mathrm{N}$. For most of the experiments in this study, a strong and deep initial vortex based on Hurricane Fran (2 Sep 1996) is used. The axisymmetric surface tangential wind distribution of the vortex is shown by the solid line in Fig. 1. We thus are starting with a hurricane of given surface maximum wind and minimum pressure. Note that in this paper surface air-sea difference refers to the difference between the lowest model layer and the sea surface, surface pressure and temperature refer to values at the sea surface, while surface wind refers to values at the lowest model level. A few experiments are also performed with a large size initial vortex indicated by the dashed line in Fig. 1. Since in all experiments the hurricane model attains quasi-steady state (regarding its intensity and overall structure) in about one or two days using these vortices, it is assumed that this steady state is representative of the maximum attainable hurricane intensity for the specified environmental conditions. The time evolution of these environmental fields was evaluated and found to be very small during 3-day runs.

For most of the experiments in this study, horizontally uniform environmental fields are used. Figure 2 a shows 


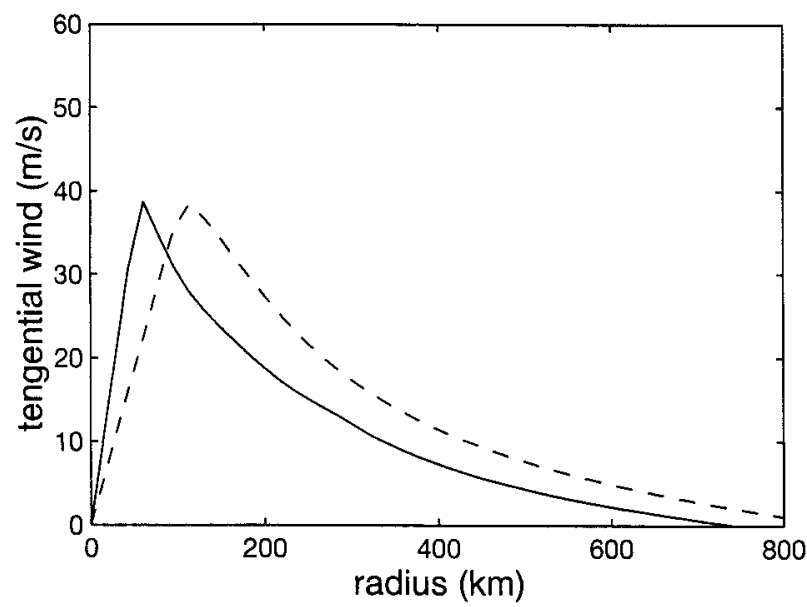

FIG. 1. Radial distributions of the surface tangential wind in the initial hurricane vortices. The initial wind distribution of the primary experiments is denoted by the solid line. The distribution used in some supplementary experiments is denoted by the dashed line.

the reference profiles of temperature and relative humidity based on the Global Atmospheric Research Program Atlantic Tropical Experiment (GATE) III conditions at $80^{\circ} \mathrm{W} .{ }^{1}$ These reference profiles are used in the control case with an underlying SST of $28.5^{\circ} \mathrm{C}$. The environmental surface pressure is set to be $1010 \mathrm{mb}$ for all experiments in this study. The temperature anomalies (from $-4^{\circ}$ to $+4^{\circ} \mathrm{C}$ in the upper troposphere) in Fig. $2 \mathrm{~b}$ are those applied to the reference temperature profile to investigate the effects of temperature lapse rate changes. Note that the opposite temperature anomalies in the stratosphere are based on the effect of increased $\mathrm{CO}_{2}$ (e.g., Hansen et al. 1984; Manabe and Wetherald 1987); these stratospheric anomalies are found to have negligible influences on the final hurricane intensities in this study. Separate experiments are performed for a range of SSTs $\left(26^{\circ}-31^{\circ} \mathrm{C}\right)$ with an increment of $0.5^{\circ} \mathrm{C}$ and for each temperature anomaly shown in Fig. 2. The same surface air-sea temperature difference of $1.2^{\circ} \mathrm{C}$ and relative humidity profile are used in most of the experiments in this study. The same relative humidity profile is used because climate model simulations reveal little variation in tropical relative humidity in global warming scenarios. Although the SST range from $26^{\circ}$ to $31^{\circ} \mathrm{C}$ may exceed the temporal variability of SST at a specific location, it fairly represents a combination of temporal and spatial variabilities in the tropical and subtropical SSTs due to doubling $\mathrm{CO}_{2}$. The $+4{ }^{\circ} \mathrm{C}$ uppertropospheric temperature anomaly relative to surface temperature is only a little larger than the maximum change indicated by climate models for doubling $\mathrm{CO}_{2}$,

\footnotetext{
${ }^{1}$ The humidity for GATE III at $80^{\circ} \mathrm{W}$ is high to a large depth compared with the moisture at other longitudes and other periods of GATE (Kurihara and Tuleya 1981).
}

while the $-4^{\circ} \mathrm{C}$ anomaly may be somewhat larger than the natural variabilities implied by Oort (1983) and Henderson-Sellers et al. (1998). This will be further discussed in the next section.

Experiments are also performed using an easterly mean wind of constant angular velocity that yields -5 $\mathrm{m} \mathrm{s}^{-1}$ at $19.5^{\circ} \mathrm{N}$ where the initial hurricane vortexes are placed. In the presence of the initial mean winds, meridional gradients of surface pressure and air temperature are obtained by solving the geostrophic wind and hydrostatic balance equations. Additional coupled hurricane-ocean experiments are also performed with this mean flow condition. In these coupled experiments, idealized ocean initial conditions are used. A resting horizontally uniform ocean is assumed of a typical vertical temperature profile in the western Atlantic Ocean in August. It has a vertically uniform mixed layer of 30-m depth with a $0.1^{\circ} \mathrm{C} \mathrm{m}^{-1}$ vertical temperature gradient in the upper thermocline layer from 30 to $100 \mathrm{~m}$ and a $0.037^{\circ} \mathrm{C} \mathrm{m}^{-1}$ gradient in the lower thermocline layer from 100 to $375 \mathrm{~m}$. The ocean depth is set to be 4000 $\mathrm{m}$ everywhere. Open ocean boundary conditions are used. The resolution of the ocean model is the same as the resolution for the most inner mesh of the atmospheric hurricane model.

\section{The results}

As stated previously, 3-day integrations are made starting with a specified vortex initialized on an idealized environmental field. Figure 3 shows time evolutions of hurricane intensity (minimum surface pressure in the upper panel and maximum surface wind in the lower panel) for different lapse rate anomalies with no mean flow. A quasi steady state is achieved after about one or two days. The cases with more unstable (stable) lapse rates tend to attain higher (lower) hurricane intensity. Changes of temperature lapse rate due to uppertropospheric temperature anomalies ranging from $2.5^{\circ}$ to $-2.5^{\circ} \mathrm{C}$ lead to hurricane minimum surface pressure changes by about $15 \mathrm{mb}$ and surface maximum wind changes by about $8 \mathrm{~m} \mathrm{~s}^{-1}$. It was found in these experiments that the sizes of the resulting hurricane and its eye are almost the same as their initial sizes with the warm core correspondingly more intense for higher hurricane intensity. Further details of hurricane structure are not investigated but a simple discussion about the most salient feature regarding the warm core intensity will be given in the next section based on the initial environmental and the simulated eyewall thermodynamic profiles.

It is important to examine whether the lapse rate differences in the above cases cause any differences in hurricane track and propagation that may be responsible, in part, for the observed differences in hurricane intensity. It was found that the hurricane track differences are small and their latitudinal and longitudinal differences at any time during the model integrations are 
(a) Temperature/relative humidity

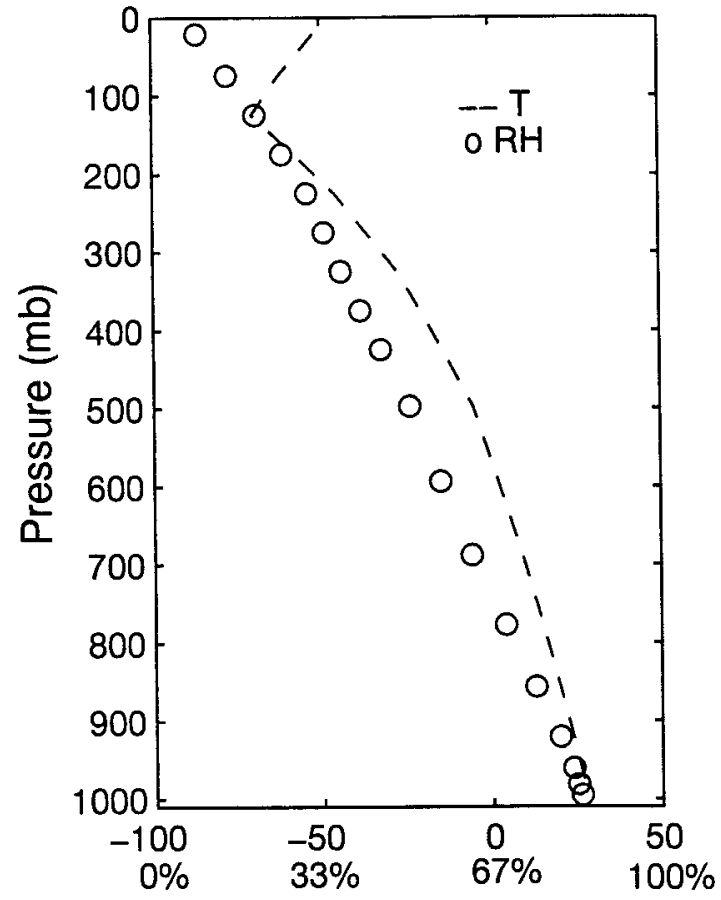

(b)

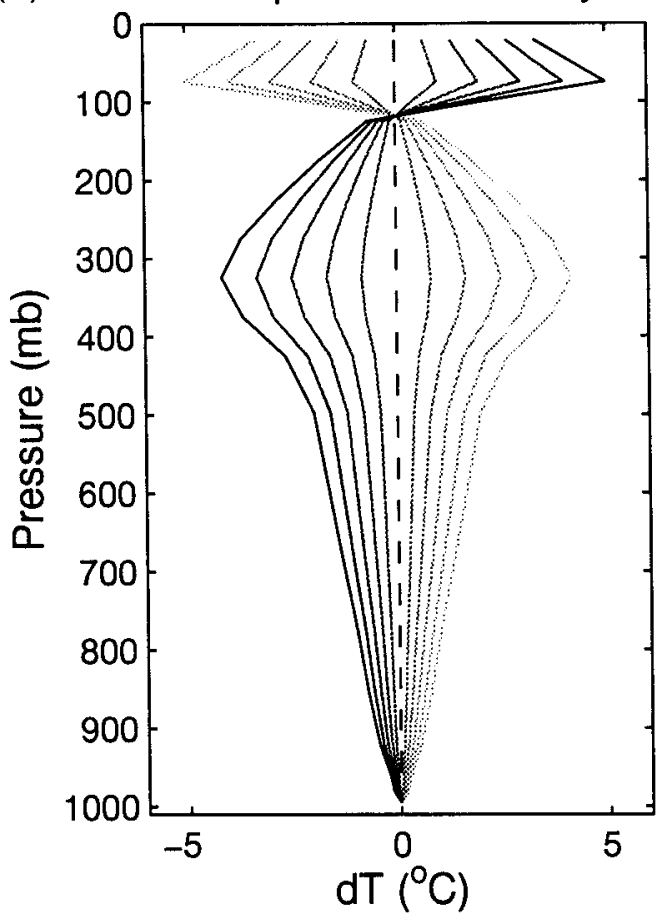

FIG. 2. (a) Vertical distributions of temperature (dashed line) and relative humidity (circles) used in the control case with SST of $28.5^{\circ} \mathrm{C}$. The surface air-sea temperature difference is $1.2^{\circ} \mathrm{C}$. Surface relative humidity is $84 \%$. In the cases with different SSTs and no lapse rate anomaly, the same surface air-sea temperature difference and the lapse rate, $-d T / d z$, profile of this temperature distribution are used. (b) Temperature anomalies used in the experiments.

smaller than $0.5^{\circ}$. Other experiments were also performed with initial position perturbed up to $0.5^{\circ}$ for the same lapse rate conditions. The results indicated that compared to the intensity difference shown in Fig. 3 the intensity difference due to the track difference caused by the initial position perturbation is negligible. Hence, any influence from the track differences on hurricane intensity difference should be negligible in the present cases. It should be pointed out that any temporal changes of the hurricane environment from its initial state are found to be small during the 3-day integrations. We have examined the temporal evolution of the environmental lapse rates, CAPEs, and the surface air-sea entropy differences. It was found that their variation during 3-day integrations is small and the differences in these quantities among the experiments during the 3-day integrations are quite consistent with their initial differences.

The third day (48-72 h) averaged minimum surface pressure is used to represent the model-attained maximum intensity (hereafter referred to as hurricane intensity). The result of the hurricane intensity as a function of environmental lapse rate and SST is given in Fig. 4. The offsetting relationship between the effects of lapse rate decrease (or stability increase) and SST increase is monotonic and quite systematic in the parameter space investigated. For example, the effect of an SST increase of $1.5^{\circ} \mathrm{C}$ can be compensated by the effect of a positive upper-tropospheric temperature anomaly of about $3^{\circ}-$ $4^{\circ} \mathrm{C}$. Another feature in this figure is that the effect of the same SST or lapse rate change is lager with higher SSTs and more unstable lapse rates (lower-right corner of Fig. 4) than with lower SSTs and more stable lapse rates (upper-left corner). This implies that more intense hurricanes have larger intensity changes given the same magnitude change in temperature lapse rate or SST. Most climate models indicate that an increase of SST of about $2^{\circ} \mathrm{C}$ is accompanied by an upper-tropospheric temperature anomaly of about $1.5^{\circ}-4^{\circ} \mathrm{C}$ for an increased $\mathrm{CO}_{2}$ effect (e.g., Henderson-Sellers et al. 1998). Thus, a typical $\mathrm{CO}_{2}$-induced warming will lead to a modelattained intensity increase about $7-8 \mathrm{mb}$ if an SST of $28.5^{\circ}$ with the control lapse rate profile is considered the current climate. Without the stabilization aloft, the attained intensity increase would nearly double. These results agree with those of Henderson-Sellers et al. (1998) and Knutson et al. (1998) who determined cyclone intensities based on estimated climate change statistics from the GFDL climate model.

In this idealized investigation, the upper-level temperature anomalies and SSTs were specified rather arbitrarily. In reality, however, static stability and SST are correlated. As an estimate for the observed temporal and spacial variability, concurrent SSTs and upper-level temperature anomalies were calculated from the GFDL 
(a)

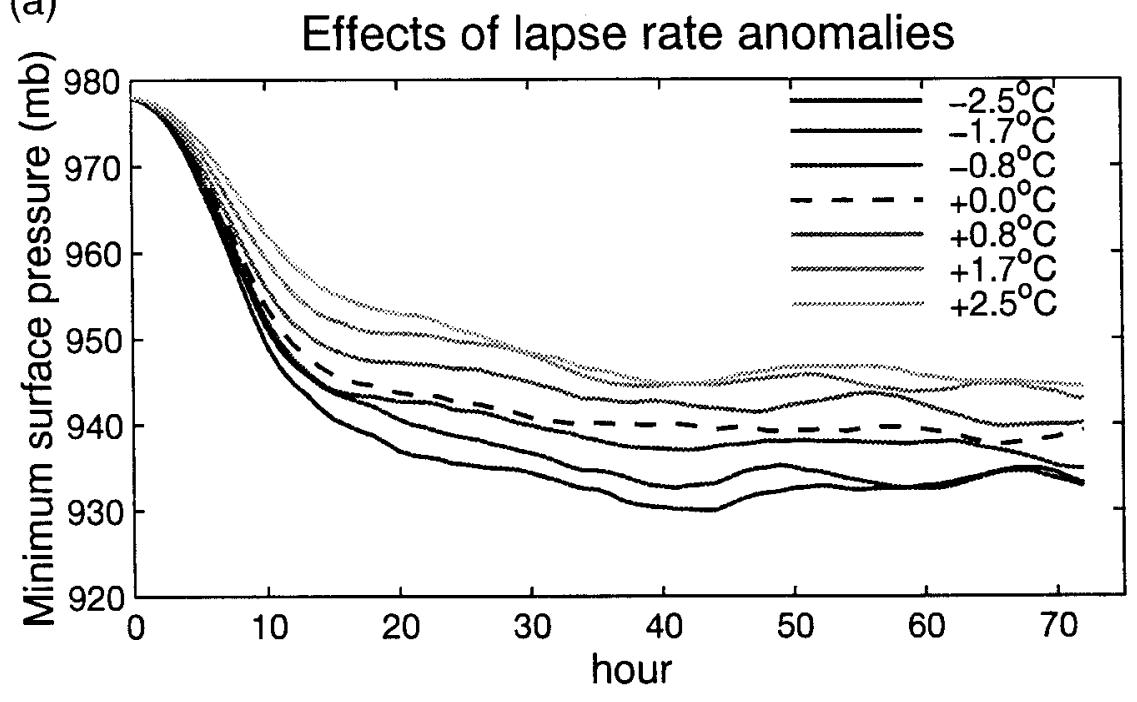

(b)

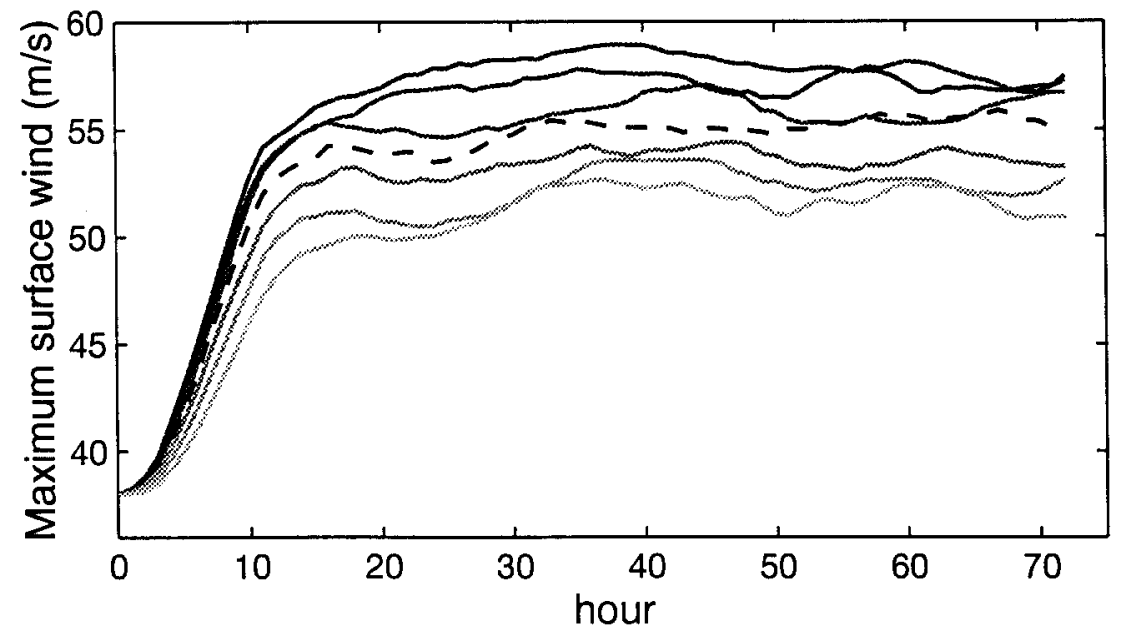

FIG. 3. Intensity evolution, indicated by (a) the minimum surface pressure and (b) maximum surface wind, during model integrations for seven cases of different lapse rate anomalies with no environmental wind. SST is $28.5^{\circ} \mathrm{C}$ in these cases. Temperature lapse rates destabilize with increasing darkness of the intensity lines but with the reference state dashed. The numbers from $-2.5^{\circ} \mathrm{C}$ to $+2.5^{\circ} \mathrm{C}$ in the upper right represent the maximum temperature anomalies in the upper troposphere shown in Fig. $2 \mathrm{~b}$.

climate model simulations. The calculations were made for all ocean locations between $10^{\circ}$ and $30^{\circ} \mathrm{N}$ for the $1^{\circ}$ $\times 1^{\circ}$ initial fields, which were used in the experiments of Knutson et al. (1998). The dots in Fig. 4 show the upper-tropospheric temperature anomalies for different SSTs with the surface relative humidity of $83 \%-85 \%$. The dark (light) dots denote those in the current (high) $\mathrm{CO}_{2}$ climate model simulations. The upper-tropospheric temperature anomalies related to the dots are the deviations of $T_{320 \mathrm{mb}}-T_{1000 \mathrm{mb}}$ from the mean value in the current climate. The individual climate values show considerable spread with high- $\mathrm{CO}_{2}$ values generally at higher SSTs and larger positive temperature anomalies than control values. This indicates that most parts of the parameter space may be attainable, although some parts are more likely at least as far as the GFDL climate model is concerned.

Experiments with an easterly $\left(-5 \mathrm{~m} \mathrm{~s}^{-1}\right)$ zonal wind in the initial environment are also performed. The experiments with the easterly zonal wind are summarized in Fig. 5. There is a systematic lower intensity (about $5 \mathrm{mb}$ higher for the minimum surface pressure) than in the cases with no environmental wind. This is consistent with Bender et al. (1993a). Nevertheless, the offsetting 


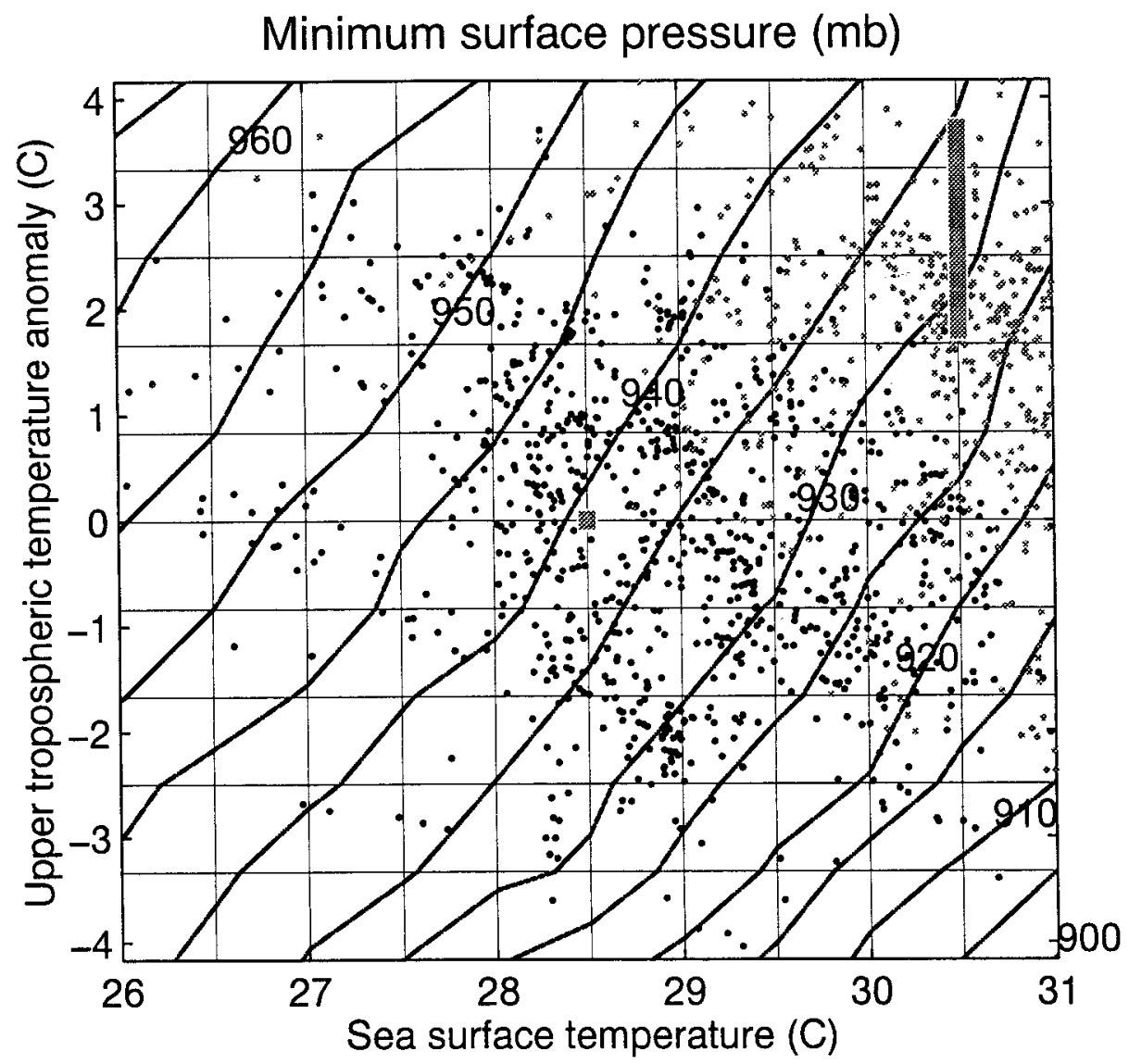

FIG. 4. Minimum surface pressures (thick lines) as a function of SST and environmental temperature lapse rate. The upper-tropospheric temperature anomaly denotes the maximum temperature anomaly in the upper troposphere relative to the surface. Positive anomalies denote stabilization. Experiments are conducted at the values of intersecting horizontal and vertical (thin) lines. The reference bar shows the range of averaged upper-tropospheric temperature increase from most climate model results related to a $2^{\circ} \mathrm{C}$ SST increase $\left(28.5^{\circ} \mathrm{C}\right.$ is used to represent the current climate in the figure) due to increased $\mathrm{CO}_{2}$ effect. The dark (light) dots denote the anomalies in the control (warmed) climate from the GFDL climate model simulations.

relationship between the effects of lapse rate decrease (increase) and SST increase (decrease) in the current cases is quite similar to that in the cases with no environmental wind. As in the no environmental wind case, the track differences among experiments with the $-5 \mathrm{~m} \mathrm{~s}^{-1}$ zonal flow are negligible. This assures that the intensity differences are nearly unrelated to the track differences in the cases with the zonal flow.

Some experiments are also performed to test the sensitivity of the results to the initial vortex wind distribution. A vortex with a different radial distribution of surface wind (the dashed line in Fig. 1) but with the same vertical variation profile and maximum surface wind is used for such a sensitivity test. Quasi steady states are also attained but more slowly (in about two days) in the current cases. Figure 6 shows the modelattained hurricane intensities with different lapse rates but a fixed SST of $28.5^{\circ} \mathrm{C}$ (thick dashed line) and with different SSTs but the control lapse rate temperature profile (thick solid line). Upper-tropospheric tempera- ture anomaly from $2.5^{\circ} \mathrm{C}$ to $-2.5^{\circ} \mathrm{C}$ and SST change from $27^{\circ}$ to $30^{\circ} \mathrm{C}$ lead to hurricane minimum surface pressure changes by about 20 and $30 \mathrm{mb}$, respectively. These changes are larger than those obtained using the Hurricane Fran (2 Sep 1996) initial vortex. However, the offsetting relationship between the lapse rate and SST effects is quite similar to that found in the previous cases. In the current cases, the radius of the maximum surface wind changes from the initial value of about 100 $\mathrm{km}$ to about $70 \mathrm{~km}$ when a quasi steady state is achieved, while with the Hurricane Fran vortex the radius of the maximum surface wind changes little from its initial value of about $55 \mathrm{~km}$. This structure adjustment is typical for initial development of most tropical cyclones and may have contributed to the longer time for the model hurricane to reach its quasi steady state. Although the characteristics of the initial vortex have an influence on the final model intensity, the difference in hurricane track and final hurricane size is very small for the performed experiments. In summary, the model-attained 


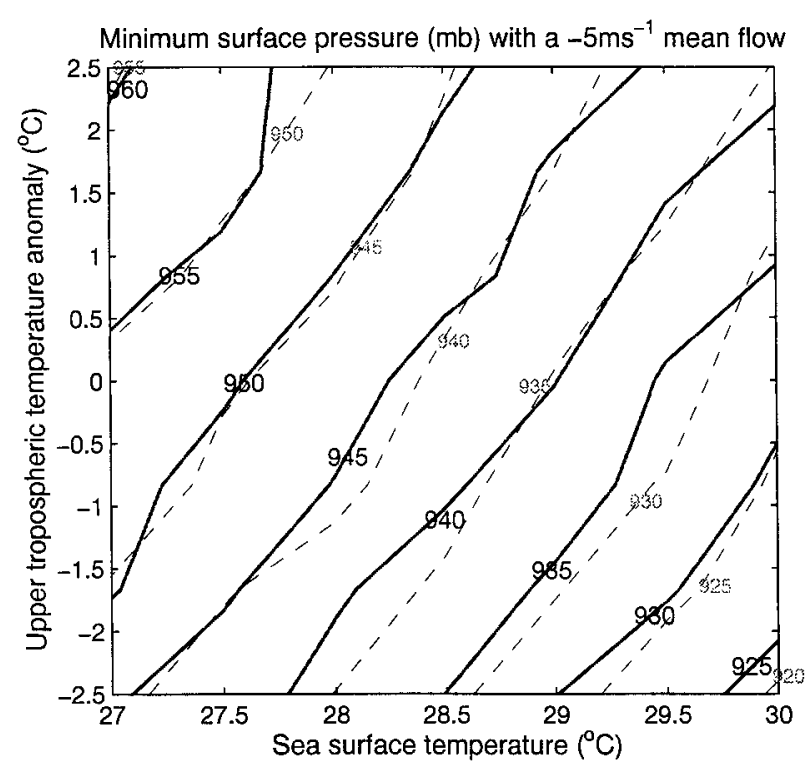

FIG. 5. Same as Fig. 4 except for a smaller parameter space with an easterly zonal wind $\left(-5 \mathrm{~m} \mathrm{~s}^{-1}\right)$ in the environment (solid line). The dashed lines denote the experimental results with no zonal wind (Fig. 4).

intensity is found to be dependent on the radial profile of the initial surface wind. Nevertheless, the zonal winds without vertical shear and the vortices used do not appreciably alter the relative effects of stability and SST changes on hurricane intensity.

Supplementary experiments have been performed to test the effects of environmental relative humidity change in the lower troposphere. Small changes $(81 \%-$ $87 \%$ ) in the surface relative humidity were used in the experiments with the same SST and lapse rate profile. Again, these small relative humidity changes were assumed to be independent of other factors. Experiments were performed both with no mean wind and with an easterly environmental zonal wind. Results (not shown) indicate little impact on hurricane intensity despite noticeable changes in the environmental CAPE.

A set of coupled hurricane-ocean experiments are also performed. An easterly mean flow of $-5 \mathrm{~m} \mathrm{~s}^{-1}$ is used in these experiments in order to introduce a moderate SST response. Note that the SST response is highly related to hurricane movement (Ginis 1995). In Fig. 7, the results of the coupled model are compared to those with fixed SST in Fig. 5. In general, stronger (weaker) hurricanes have larger (smaller) reduction in intensity due to ocean coupling. This leads to less sensitivity of hurricane intensity to atmospheric stability and SST changes. This implies a further reduction of hurricane intensity increase in the high- $\mathrm{CO}_{2}$ climate. The overall offsetting relationship between the effects of SST and stability increases on the hurricane intensity is maintained. Figure 8 shows the relationships between the intensity decrease due to ocean coupling and the hurricane intensity and between the intensity decrease and

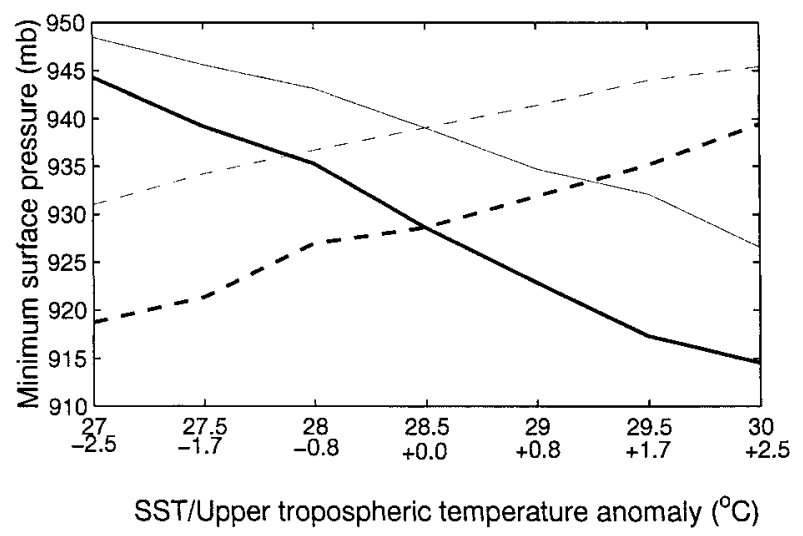

FIG. 6. The model-attained hurricane intensities for different uppertropospheric temperature anomalies with SST of $28.5^{\circ} \mathrm{C}$ (thick dashed) and for SST anomalies with the reference lapse rate profile (thick solid) in the case of the initial vortex with its radial distribution of surface wind shown by the dashed line in Fig. 1. The thin lines correspond to the smaller initial vortex (the solid line in Fig. 1).

the averaged SST cooling around hurricane center. These relationships are quite linear. ${ }^{2}$ The SST cooling from $0.4^{\circ}$ to $0.7^{\circ} \mathrm{C}$ results in minimum surface pressure increase from 1 to $9 \mathrm{mb}$. The results indicate that the hurricane intensity change depends on both its intensity and the induced SST cooling. The quasi-linear relationship between the ocean intensity decrease due to ocean

${ }^{2}$ Theoretically, the current linear relationship between the intensity and the intensity change cannot be extended to very weak hurricanes since even a very weak hurricane induces some SST cooling that leads to some intensity decrease.

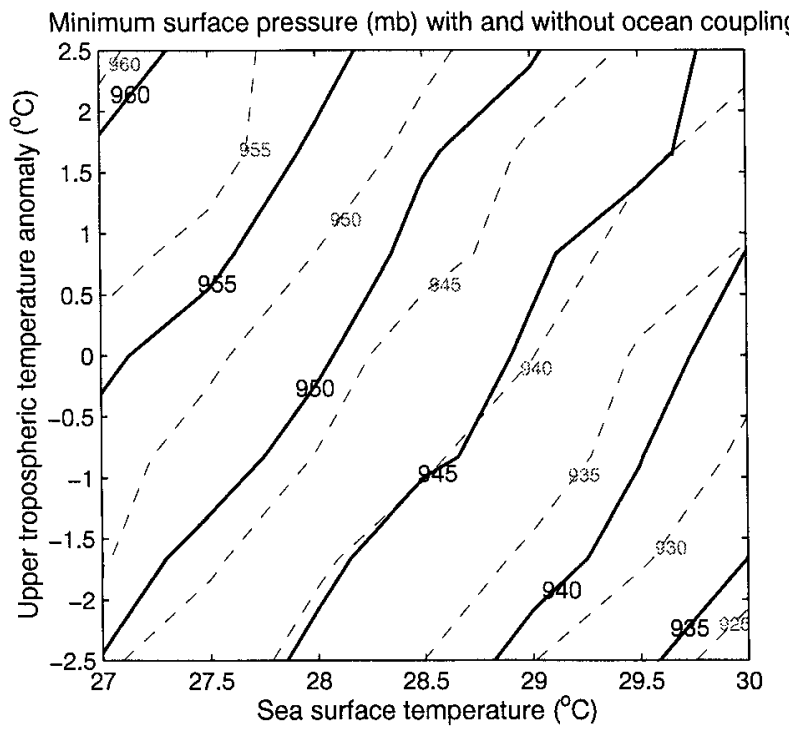

FIG. 7. Model-attained hurricane minimum surface pressures with (solid line) and without (dashed line) ocean coupling for the parameter space of Fig. 5. Values without ocean coupling are identical to the solid lines of Fig. 5. 


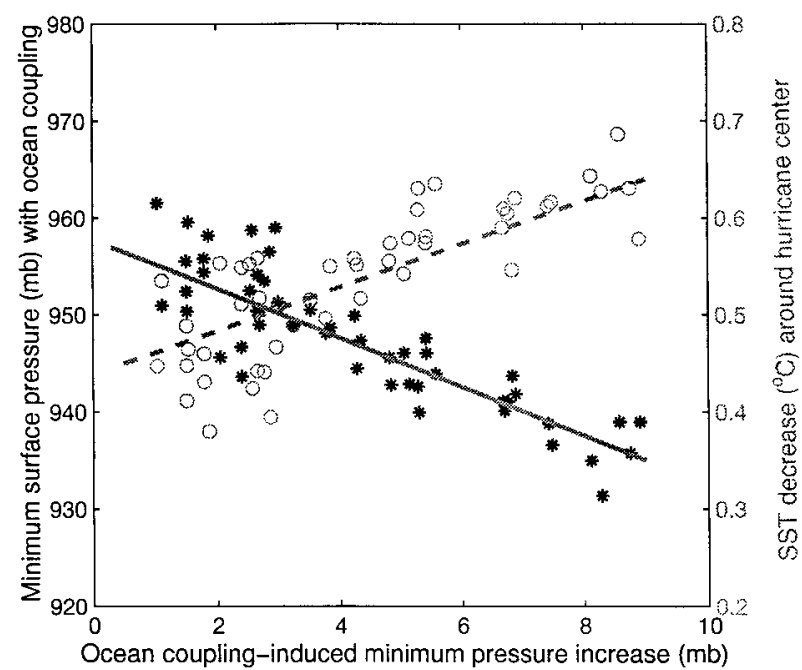

FIG. 8. The ocean coupling-induced increase of minimum surface pressure as a function of model-attained minimum surface pressures (stars with the left axis) and as a function of the averaged SST coolings around the hurricane center (radius $<100 \mathrm{~km}$, circles with the right axis).

coupling and hurricane intensity implies that the effect of ocean coupling does not alter the previous conclusion that the intensity of stronger hurricanes is more sensitive to the atmospheric stability and SST changes. Little is known about the effect of climate changes on the upper ocean structure. Further experiments with ocean coupling are needed in the future to explore the hurricane intensity changes due to variation of the ocean mixed layer and underlying temperature distribution.

\section{Discussion}

\section{a. Environmental $C A P E$}

From the results in the previous section, we can investigate the relationship between the model-attained hurricane intensity and CAPE. Since the same relative humidity profile is used, there is a unique relationship between the parameters (lapse rate and SST) and CAPE. The environmental CAPE is computed with the definition based on a surface air parcel:

$$
R_{d} \int_{p_{o}}^{p_{b}}\left(T_{p}-T_{e}\right) d \ln p,
$$

where $R_{d}$ is the gas constant, $p_{b}$ the pressure at the lowest model layer (the initial parcel level), $p_{o}$ the out-flow pressure (of neutral buoyancy), $T_{p}$ the virtual temperature of the parcel undergoing reversible-adiabatic ascent, $T_{e}$ the virtual temperature of the environment, and $p$ the pressure. Figure 9a gives the distributions of environmental CAPE and Fig. 9b the maximum surface evaporation for the lapse rates and SSTs that were used in the previous experiments. The hurricane intensity is fairly correlated with the CAPE. ${ }^{3}$ The hurricane intensity is better correlated with CAPE for the regimes with more unstable lapse rates than for the less unstable regimes. In general, an SST increase (decrease) with the same value of CAPE, which is accompanied by a less (more) unstable lapse rate, leads to an increase (decrease) of hurricane intensity. In the case of increased $\mathrm{CO}_{2}$, a $2^{\circ} \mathrm{C}$ SST increase leads to almost no CAPE change while it would be about $700 \mathrm{~J} \mathrm{~kg}^{-1}$ without the accompanying lapse rate changes. The CAPE related to the GFDL climate model simulations used by Knutson et al. (1998) was also computed (not shown). The results indicated that the climate CAPE ranges from near zero to above $5000 \mathrm{~J} \mathrm{~kg}^{-1}$ in a few simulations. The averaged CAPE, however, remains almost the same in the current and high- $\mathrm{CO}_{2}$ climates. The good relationship between the maximum surface evaporation and the hurricane intensity shown in Fig. 9b implies the importance of oceanic surface energy fluxes in determining the hurricane intensity. However, the maximum evaporation and hurricane intensity are apparently influenced by both the SSTs and upper-level temperature anomalies.

\section{b. Hurricane CAPE}

In the above discussion, the CAPE represents the potential buoyancy energy that an air parcel at the surface can gain when being lifted. Figure 10 shows various temperature profiles in the control case. The environmental CAPE is proportional to the area enclosed by the thin and thick solid lines in the figure. However, for a hurricane system, moist convection is concentrated in the eyewall whose temperature (dashed line) is much higher than the temperature of a parcel ascending from the environmental surface (thin solid line). For such a system, the energy available for hurricane maintenance may be represented by the shaded area in Fig. 10 (e.g., Riehl 1954; Emanuel 1988). Such available energy for a hurricane can be obtained by assuming an approximate wet-adiabatic profile in the eyewall (dashed line) rather than the parcel ascent of environmental air (thin solid line) in the CAPE formula, assuming the same $T_{e}$ from the environmental sounding. The so-defined CAPE will be referred to as the hurricane CAPE hereafter. It is noteworthy that if the surface environmental air should converge toward the eyewall adiabatically, the surface temperature in the eyewall would be represented by that of the thin solid line at the eyewall pressure, implying no difference between environmental CAPE, and hurricane CAPE. Thus diabatic processes mainly associated

\footnotetext{
${ }^{3}$ A pseudoadiabatic ascent would lead to larger values of CAPE but of a similar pattern in the the parameter space. A reversible ascent was chosen here because some tropical observations ( $\mathrm{Xu}$ and Emanuel 1989) indicate near-neutral buoyancy for this type of ascent. Perhaps a more realistic case should be somewhat between the pseudoadiabatic and reversible ascents.
} 
(a) Environmental CAPE $(\mathrm{J} / \mathrm{kg})$

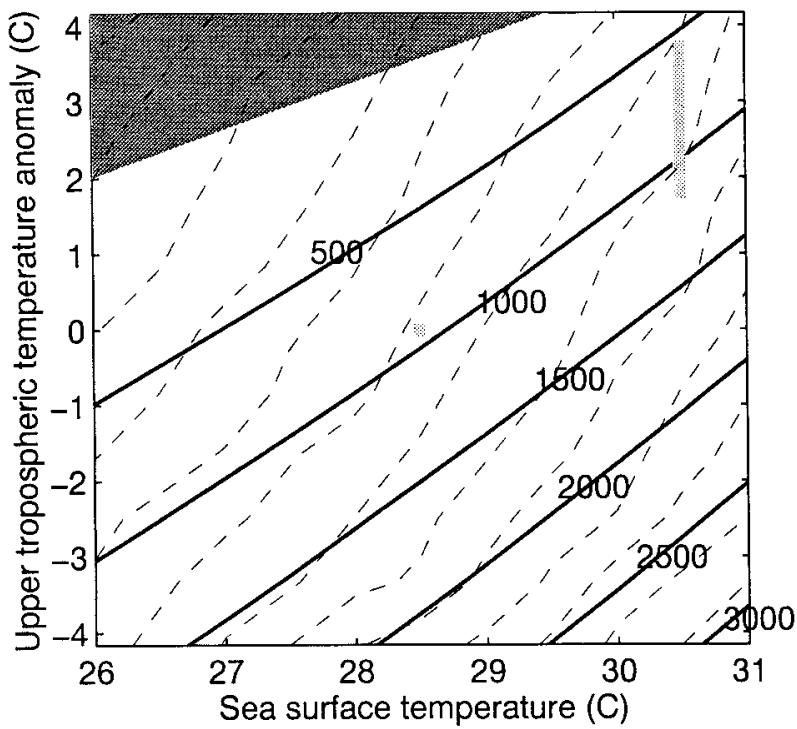

(b) Maximum evaporation ( $\mathrm{mm} / \mathrm{hr}$ )

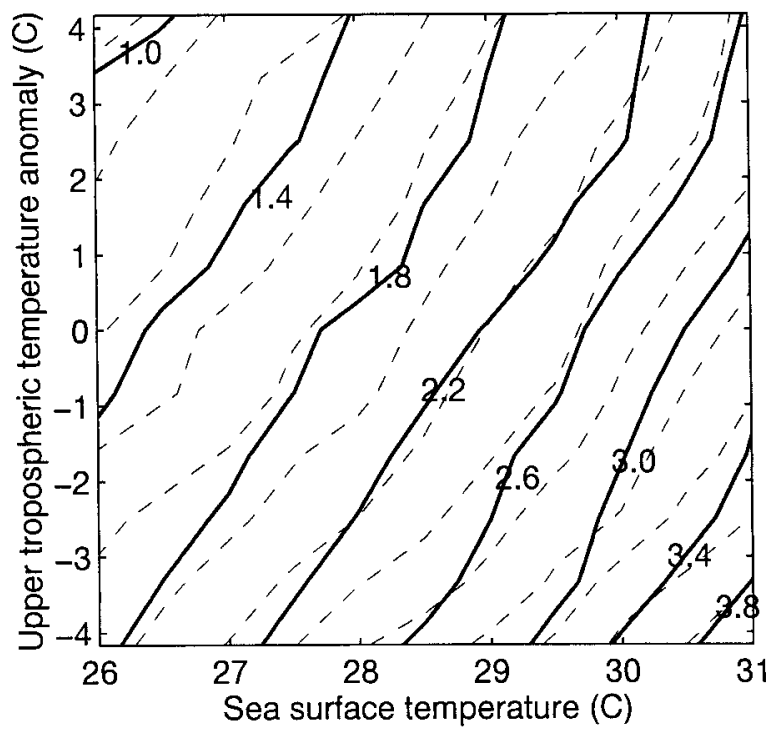

FIG. 9. (a) Initial environmental CAPE (solid lines) for reversible ascent as a function of SST and temperature lapse rate for the parameter space used in this study. The dashed contours denote the model-attained hurricane minimum surface pressures consistent with those in Fig. 4. The shading at the upper-left corner denotes no CAPE. The reference bar represents the increased $\mathrm{CO}_{2}$ condition as shown in Fig. 4. (b) Same as the upper but for maximum surface evaporation found during each experiment.

with the air-sea exchanges (Fig. 9) are very important to distinguish the hurricane CAPE from the environmental CAPE.

Figure 11 shows the hurricane CAPE as a function of environmental SST and lapse rate for the parameter space investigated. The hurricane intensity is much better correlated with hurricane CAPE than with the environmental CAPE. Furthermore, the changes of hur-

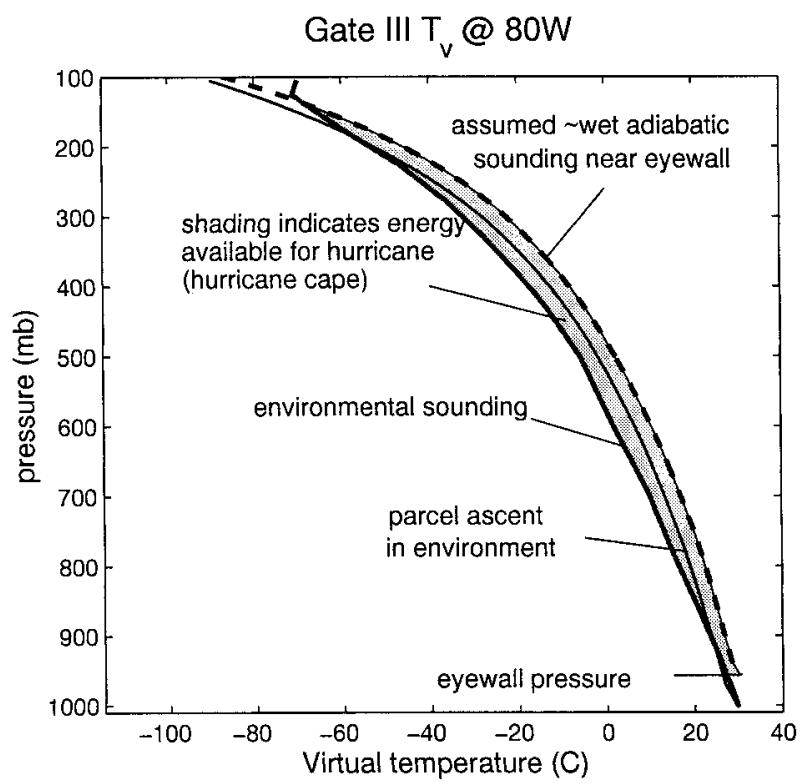

FIG. 10. Environmental sounding and wet-adiabatic profiles based on the surface environmental and eyewall parcel ascents in the control case where the surface relative humidity is $84 \%$ in the environment and $90 \%$ in the eyewall for SST of $28.5^{\circ} \mathrm{C}$ and no upper-level temperature anomaly.

ricane CAPE associated with the same SST or lapse rate change are larger for higher SSTs and more unstable lapse rates than for lower SSTs and more stable lapse rates. This seems to be consistent with the larger modelattained intensity change for higher SSTs and more un-

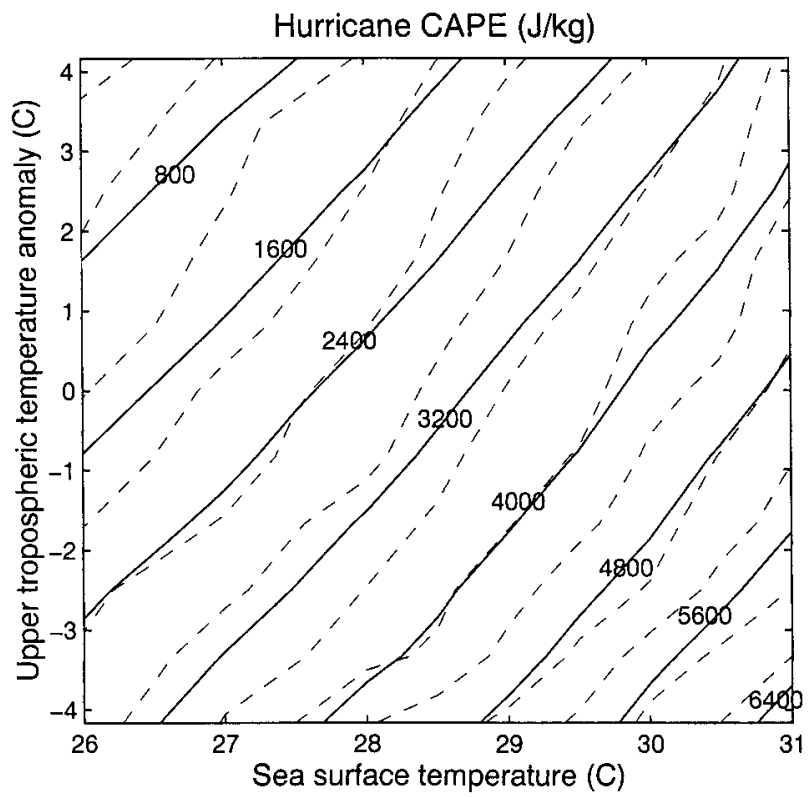

FIG. 11. Hurricane CAPE as a function of environmental SST and temperature lapse rate. The thin dashed contours indicate the modelattained minimum surface pressures of Fig. 4. Quasi-steady model estimates of surface pressure and relative humidity in the eyewall are used to calculate this hurricane CAPE. 
stable lapse rates. Note that surface relative humidity of $90 \%$ and surface air temperature of $1.5^{\circ} \mathrm{C}$ less than the SST in the eyewall were used in the hurricane CAPE calculations. These values are representative of those attained in the model experiments. The small differences of these values from case to case were found to have an insignificant influence on the value and pattern of the hurricane CAPE. Calculations using the same surface pressure in the eyewall as that in the environment indicate that about $30 \%-40 \%$ of the change from environmental CAPE to hurricane CAPE in Fig. 11 is due to the surface pressure change. The CAPE from these calculations also indicates a pattern similar to that in Fig. 11. This is consistent with the high correlation between the hurricane intensity and CAPE $(\mathrm{RH}=90 \%)$ in Knutson and Tuleya (1999).

It is found that the hurricane intensity and hurricane CAPE are also well correlated in the case with surface relative humidity changes, while the relationship between the hurricane intensity and environmental CAPE collapses. This may be because the thermodynamic properties of the surface air parcel near the eyewall depend on both air-sea energy flux and advection of the surrounding air. Apparently, environmental CAPE is not a requirement for hurricane maintenence (Fig. 9a, upper left). However, the existence of environmental CAPE appears to enhance model hurricane intensity.

It is interesting that although a $1.2^{\circ} \mathrm{C}$ surface air-sea temperature difference is initially specified for the environment in the experiments, the surface air of a mature storm particularly of high intensity is found to have a temperature quite close to the SST in the outer region (radii from about 150 to $400 \mathrm{~km}$ ). However, the surface air temperature decreases when approaching the eyewall. Observational evidence (e.g., Beckerle 1974; Cione and Black 1998) also points to an enhanced airsea temperature difference near the hurricane center. Due to the diabatic exchanges in the experiments, the total surface temperature decrease from the outer region to the eyewall is smaller than that by an adiabatic thermal expansion (e.g., the temperature decrease is $1.5^{\circ} \mathrm{C}$ in the control case while the decrease by an adiabatic thermal expansion is $2.5^{\circ} \mathrm{C}$ ).

\section{c. Effects of environmental soundings and SSTs on the warm core}

For fixed SSTs, parcel theory indicates that more unstable lapse rate produces larger eyewall-environment temperature difference and hurricane CAPE, assuming the same wet-adiabatic profile in the eyewall. This can be clearly shown using Fig. 10 when the environmental temperatures aloft are colder (more unstable). With the inclusion of the simulated lower surface pressure near the eyewall for more unstable lapse rates, the eyewallenvironment temperature difference becomes even larger. Although the model-simulated surface relative humidity, surface temperature, and eyewall sounding vary

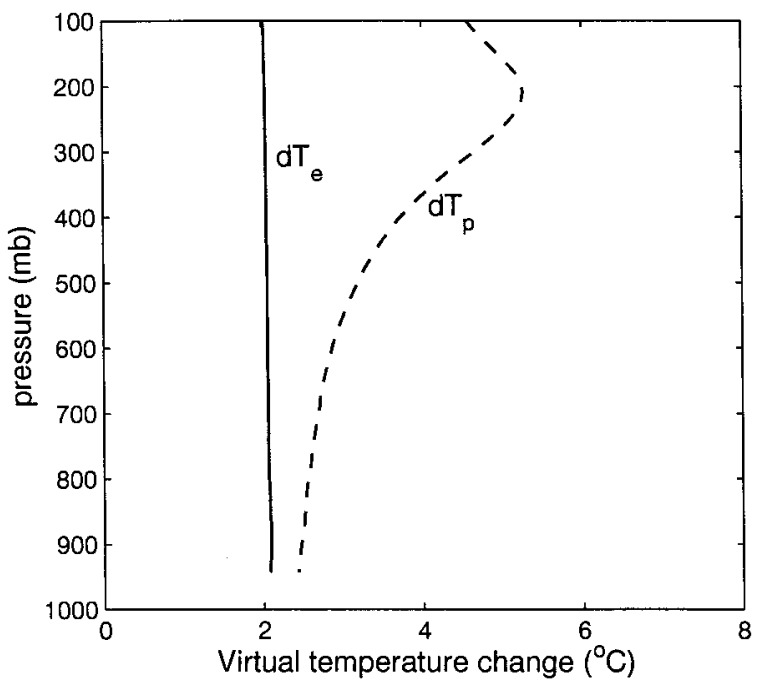

FIG. 12. Vertical distribution of the virtual temperature changes due to a $2^{\circ} \mathrm{C} \mathrm{SST}$ increase relative to the case shown in Fig. 10. Here $d T_{e}$ is the change of environmental sounding temperature; $\mathrm{d} T_{p}$ is the change of eyewall parcel temperature. Same environmental relative humidity and lapse rate profiles but with a $2^{\circ} \mathrm{C}$ surface air temperature increase are used for the solid line. Same surface relative humidity and pressure in the eyewall but with a $2^{\circ} \mathrm{C}$ surface air temperature increase are used for the dashed line.

from case to case, these variations appear to be small. In the model experiments with different lapse rate profiles, the eyewall-environment temperature difference was found to be mainly determined by the environmental sounding difference. This may explain why more intense warm cores were found to be associated with the cases of more unstable lapse rates.

For the experiments with different SSTs but with the same lapse rate profile, the difference in the eyewall wet adiabat may be used to explain the intensity of the warm core and, thus, the storm intensity. Figure 12 shows the changes of environmental and eyewall virtual temperatures due to a $2^{\circ} \mathrm{C}$ surface temperature increase relative to the reference case in Fig. 10 but with the same surface pressure and relative humidity in the eyewall. The increase of hurricane CAPE is proportional to the area between the dashed and solid lines below the outflow layer (about $100 \mathrm{mb}$ in this case). It is clear that the hurricane CAPE increase with increased surface temperature is due to the nonlinear change of the eyewall sounding (i.e., the wet adiabat). The inclusion of the simulated decrease in surface pressure near the eyewall in the higher SST cases moves the dashed line (in Fig. 12) farther right. As in the cases of different lapse rate profiles, the model-simulated surface relative humidity, surface air temperature, and eyewall sounding vary from case to case if SST is different, but such differences were found to only have minor modifications to the calculated wet-adiabatic ascent (dashed line in Fig. 12). Thus, the large temperature increase in the eyewall for an SST increase explains the larger environment-eyewall temperature difference and the more intense warm 


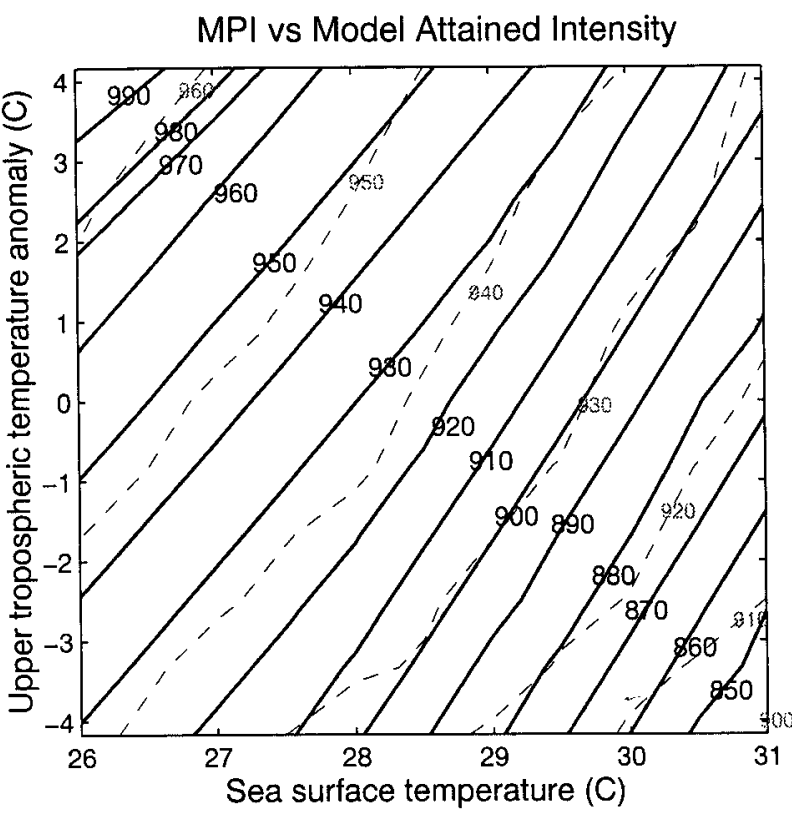

FIG. 13. Maximum potential intensity (solid) calculated by the Holland (1997) thermodynamic approach for the parameter space of the present study and the model-attained intensity (dashed) of Fig. 4.

core (not shown). Since the largest eyewall temperature increase (around $200 \mathrm{mb}$ ) is located above the the warm core center (around $300 \mathrm{mb}$ ), this may also explain why higher (in altitude) warm cores have been found with higher SSTs in the experiments (not shown).

In summary, changes of initial environmental lapse rate affect hurricane CAPE, warm core intensity, and thus hurricane intensity mainly by changing the environmental temperature aloft (the thick solid line in Fig. 10). On the other hand, changes of SST affect hurricane CAPE, warm core intensity, and hurricane intensity mainly by changing the slope of the eyewall wet-adiabatic ascent (the dashed line in Fig. 10).

\section{d. Comparison with MPI theories}

It is interesting to compare the present results with MPI theories for the parameter space investigated. Figure 13 shows the Holland (1997) MPI (solid) in comparison with the model-attained maximum intensity (dashed). The patterns are quite similar. In general, however, the GFDL model intensity is less sensitive to the SST and lapse rate changes than the Holland (1997) MPI. The Holland approach also predicts larger (smaller) intensity change for stronger (weaker) hurricanes given the same magnitude change in SST and temperature lapse rate. The abrupt intensity change around 970 $\mathrm{mb}$ by the Holland approach is due to an intensity criterion used in which the eyewall pressure is used for the minimum surface pressure for weak storms. For the low SSTs and high stability regimes in the upper-left corner of the parameter space (Fig. 9), the GFDL model- attained hurricane intensity may have been influenced by the rather strong initial vortex used. Nevertheless, Fig. 10 indicates that a hurricane can be maintained in this neutral or stable state if an initial low-pressure vortex preexists. Due to the existence of CAPE and its large variation in the present study, the GFDL model intensity pattern in Fig. 4 somewhat departs from those by the MPI approach of Emanuel (1995), which is relatively CAPE insensitive. Nevertheless, as pointed out by Knutson et al. (1998), the GFDL model yields similar intensity tendencies to the MPI estimates of Holland and Emanuel for the global change scenarios in which SST increases are accompanied by more unstable lapse rates.

\section{Summary}

The GFDL hurricane model has been used to study the sensitivity of hurricane intensity to thermodynamic environment for a wide range of upper-tropospheric temperature anomalies and SST changes. With the same relative humidity profile, this yields atmospheric CAPE of a broad spectrum from zero to about $3000 \mathrm{~J} \mathrm{~kg}^{-1}$ for the parameter space investigated. A few experiments were also performed with ocean coupling. The main results of this study are summarized as follows.

1) The results show that stabilization in the environmental atmosphere and SST increase cause opposing effects on hurricane intensity. Any potential hurricane intensity change due to SST increase of $1.5^{\circ} \mathrm{C}$ can be compensated by an atmospheric stabilization due to upper tropical tropospheric temperature increase of about $3^{\circ}-4^{\circ} \mathrm{C}$ relative to the surface temperature. The offsetting relationship between the effects of stabilization (destabilization) and SST increase (decrease) is monotonic and quite systematic in the parameter space investigated $\left(26^{\circ}-31^{\circ} \mathrm{C}\right.$ for SST and upper-tropospheric temperature anomalies of $\pm 4{ }^{\circ} \mathrm{C}$ relative to a control profile). This offsetting relationship appears relatively independent of the zonal environmental wind without shear, the hurricane size, and the surface wind profile of the initial vortex. The results imply that any hurricane intensity increase related to a possible warming due to increased $\mathrm{CO}_{2}$ is much smaller than that would be expected by considering SST increases alone.

2) Hurricane intensity is more sensitive to lapse rate and SST changes over a warmer region with a more unstable lapse rate than over a colder region with a less unstable lapse rate for the parameter space investigated. Since the model attains higher intensities over warmer regions with more unstable lapse rates, this result is equivalent to the fact that more intense hurricanes are more sensitive to temperature lapse rate and SST changes.

3) Inclusion of ocean coupling leads to reduction of hurricane intensity but the intensity reduction of a stronger hurricane is larger than that of a weak hur- 
ricane. This is because a stronger hurricane generates more vigorous mixing of the ocean mixed layer and thus larger sea surface cooling, which reduces hurricane intensity. On the other hand, the ocean coupling does not change the overall offsetting relationship between the effects of atmospheric stabilization and SST increases. This implies less sensitivity of hurricane intensity to any given changes of environmental stability and SST with ocean coupling than without ocean coupling, and thus less hurricane intensity increase in the high- $\mathrm{CO}_{2}$ climate.

4) Hurricane intensity is correlated with the environmental CAPE at least for the experiments where the initial relative humidity is fixed. However, in general, an SST increase (decrease) with the same value of CAPE, which is accompanied by a less (more) unstable lapse rate, leads to an increase (decrease) of hurricane intensity. The hurricane intensity is also well correlated with the maximum surface evaporation in the experiments of lapse rate and SST changes. The hurricane intensity is found to be very highly correlated with the "hurricane CAPE," defined as the energy available from wet-adiabatic ascent in the eyewall relative to a reference sounding in the undisturbed environment. Hurricane CAPEs of high values are found to be more sensitive to lapse rate and SST changes, which is consistent with the result that more intense hurricanes are more sensitive to temperature lapse rate and SST changes. A strong relationship between hurricane intensity and hurricane CAPE also applies to the cases with small surface relative humidity changes where the relationship between hurricane intensity and environmental CAPE collapses. Since hurricane CAPE compares the eyewall and environmental thermodynamic profiles, the surface diabatic exchanges are indirectly considered. The better representativeness of this CAPE can be considered a combination index of atmospheric and oceanic influences.

5) The difference of warm core intensity for different initial lapse rates is mainly due to the environmental sounding differences while differences of the eyewall sounding appear to be small. In the cases of SST differences, the warm cores are more intense and higher (in altitude) with more intense hurricanes due to higher SSTs. It is found that in these cases, the nonlinear change of the eyewall sounding due to SST change is very important. The diagnosis of the position of the largest eyewall temperature increase (around $200 \mathrm{mb}$ ) using parcel theory may also explain the upward movement of the warm core for the cases of higher SSTs.

The results in this study were obtained with numerical experiments using the GFDL hurricane model. It should be pointed out that the operational GFDL hurricane model intensity prediction has not been as successful as its track prediction. The intensity prediction error is like- ly related to the current model resolution, the treatment of deep convection in the model, and probably the current initialization scheme in the operational forecasts (Kurihara et al. 1998). However, in this idealized study with a focus on maximum achievable model hurricane intensities with specified environment and vortex conditions, it is anticipated that model variations in intensity may be more reliable than those found in operational forecasts.

Acknowledgments. The authors appreciate the continuous encouragement and very helpful comments by Yoshio Kurihara and Jerry Malhman. They also wish to thank Tom Knutson and Morris Bender for their useful discussions and communications during the course of this study. Programming assistance in setting the ocean model from Clark Rowley is appreciated. This work was supported in part by the Risk Prediction Initiative Program under the auspices of the Atlantic Global Change program. Computer resources were supplied by the GFDL computer facilities.

\section{REFERENCES}

Beckerle, J. C., 1974: Air and sea temperatures during traverse of hurricane Alma 1966. J. Phys. Oceanogr., 4, 487-492.

Bender, M. A., and I. Ginis, 2000: Real case simulations of hurricaneocean interaction using a high-resolution coupled model: Effect on hurricane intensity. Mon. Wea. Rev., in press.

,$- \ldots$, and Y. Kurihara, 1993a: Numerical simulations of tropical cyclone-ocean interaction with a high-resolution coupled model. J. Geopys. Res., 98 (D12), 23 245-23 262.

_ R. J. Ross, R. E. Tuleya, and Y. Kurihara, 1993b: Improvements in tropical cyclone track and intensity forecasts using the GFDL initialization system. Mon. Wea. Rev., 121, 2047-2061.

Betts, A. K., 1982: Saturation point analysis of moist convective overturning. J. Atmos. Sci., 39, 674-701.

Blumberg, A. F., and G. L. Mellor, 1987: A description of a threedimensional coastal ocean circulation model. Three-Dimensional Coastal Ocean Models, N. Heaps, Ed., American Geophysical Union, $208 \mathrm{pp}$.

Cione, J. J., and P. G. Black, 1998: Surface thermodynamic observations within the tropical cyclone inner core. Preprints, Symp. on Tropical Cyclone Intensity Change, Phoenix, AZ, Amer. Meteor. Soc., 141-145.

Emanuel, K. A., 1987: The dependence of hurricane intensity on climate. Nature, 326, 483-485.

, 1988: Toward a general theory of hurricanes. Amer. Sci., 76, 371-379.

_ 1995: Sensitivity of tropical cyclones to surface exchange coefficients and a revised steady-state model incorporating eye dynamics. J. Atmos. Sci., 52, 3969-3976.

— J. D. Neelin, and C. S. Bretherton, 1994: On large scale circulations in convecting atmospheres. Quart. J. Roy. Meteor. Soc., 120, 1111-1143.

Ginis, I., 1995: Ocean response to tropical cyclones. Global Perspectives on Tropical Cyclones, R. Elsberry, Ed., World Meteorological Organization., 198-260.

Hansen, J., A. Lacis, D. Rind, L. Russell, P. Stone, I. Fung, R. Ruedy, and J. Lerner, 1984: Climate sensitivity analysis of feedback mechanisms. Climate Processes and Climate Sensitivity, Geophys. Monogr., No. 29, Amer. Geophys. Union, 130-163.

Henderson-Sellers, A., and Coauthors, 1998: Tropical cyclones and global climate change: A post-IPCC assessment. Bull. Amer. Meteor. Soc., 79, 19-38. 
Holland, J. G., 1997: The maximum potential intensity of tropical cyclones. J. Atmos. Sci., 54, 2519-2540.

Knutson, T. R., and R. E. Tuleya, 1999: Increased hurricane intensity with $\mathrm{CO}_{2}$-induced warming as simulated using the GFDL hurricane prediction system. Climate Dyn., 15, 503-519.

,$- \ldots$, and Y. Kurihara, 1998: Simulated increase of hurricane intensities in a $\mathrm{CO}_{2}$-warmed climate. Science, 279, 1018-1020.

Kurihara, Y., 1973: A scheme of moist convective adjustment. Mon. Wea. Rev., 101, 547-553.

_ for tracking a small vortex. Mon. Wea. Rev., 108, 1792-1809.

_ , and R. E. Tuleya, 1981: A numerical simulation study on the genesis of a tropical storm. Mon. Wea. Rev., 109, 1629-1653.

— experiments of Hurricane Gloria (1985) using a multiply nested movable mesh model. Mon. Wea. Rev., 118, 2185-2198.

- - - , and R. J. Ross, 1993: An initialization scheme of hurricane models by vortex specification. Mon. Wea. Rev., 121, 2030-2045.

,-- , R. E. Tuleya, and R. J. Ross, 1995: Improvements in the GFDL hurricane prediction system. Mon. Wea. Rev., 123, 27912801.

- , R. E. Tuleya, and M. A. Bender, 1998: The GFDL hurricane prediction system and its performance in the 1995 hurricane season. Mon. Wea. Rev., 126, 1306-1322.

Lacis, A. A., and J. E. Hansen, 1974: A parameterization for the absorption of solar radiation in the earth's atmosphere. J. Atmos. Sci., 31, 118-133.
Lighthill, J., and Coauthors, 1994: Global climate change and tropical cyclones. Bull. Amer. Meteor. Soc., 75, 2147-2157.

Manabe, S., and R. T. Wetherald, 1987: Tropical circulation in a time integration of a global model of soil wetness induced by an increase in atmospheric Carbon dioxide. J. Atmos. Sci., 44, 12111235.

Mellor, G. L., and T. Yamada, 1974: A hierarchy of turbulence closure models for planetary boundary layers. J. Atmos. Sci., 31, 17911806.

Oort, A. H., 1983: Global atmospheric circulation statistics, 19581983. NOAA Prof. Paper 14, $180 \mathrm{pp}$

Riehl, H., 1954: Tropical Meteorology. McGraw-Hill, 392 pp.

Schade, L. R., and K. A. Emanual, 1999: The ocean's effect on the intensity of tropical cyclones: Results from a simple coupled atmosphere-ocean model. J. Atmos. Sci., 56, 642-651.

Schwarzkopf, M. D., and S. B. Fels, 1991: The simplified exchange method revised: An accurate, rapid method for computation of infrared cooling rates and fluxes. J. Geophys. Res., 96, 90759096.

Smagorinsky, J., 1963: General circulation experiments with the primitive equations. I. The basic experiment. Mon. Wea. Rev., 91, 99-164.

Williams, E., and N. Renno, 1993: An analysis of the conditional instability of the tropical atmosphere. Mon. Wea. Rev., 121, 2136.

Xu, K.-M., and K. A. Emanuel, 1989: Is the tropical atmosphere conditionally unstable? Mon. Wea. Rev., 117, 1471-1479.

Ye, B., A. O. Genio, and K.-W. Lo, 1998: CAPE variation in the current climate and in a climate change. J. Climate, 11, 19972015 . 